\title{
Cryoballoon ablation of atrial fibrillation: How important is the proper selection of patients?
}

\author{
Maciej Wójcik ${ }^{1,2}$, Alexander Berkowitsch ${ }^{1}$, Sergey Zaltsberg ${ }^{1}$, \\ Christian W. Hamm ${ }^{1,3}$, Heinz F. Pitschner ${ }^{1}$, Malte Kuniss ${ }^{1}$, Thomas Neumann ${ }^{1,3}$ \\ ${ }^{1}$ Department of Cardiology, Kerckhoff Heart and Thorax Center, Bad Nauheim, Germany \\ ${ }^{2}$ Department of Cardiology, Medical University of Lublin, Lublin, Poland \\ ${ }^{3}$ Department of Cardiology, Justus-Liebig University of Giessen, Germany
}

\begin{abstract}
Background: Relation between pre-procedural selection of patients and the success rate after a single cryoballoon ablation (CAB) procedure is unknown.

Methods: $C A B$ was performed in 378 (65\% male, median age 58 years, 85\% paroxysmal atrial fibrillation $[A F]$ ) consecutive patients with symptomatic and drug refractory $A F$. The combined ALARMEc (Atrial fibrillation type, Left Atrium size, Renal insufficiency, Metabolic syndrome, cardiomyopathy) risk score was calculated for each individual patient. The end-point of the study was the first AF, atrial flutter or atrial tachycardia recurrence after the 3-month blanking period in the 1-year follow-up since the index procedure, in the absence of anti-arrhythmic (class I and III) therapy.
\end{abstract}

Results: Single and multi-catheter approach was used in $79 \%$ and $21 \%$ of patients, respectively. The acute success rate with single and multi-catheter approach was 79\% and 99\%, respectively. The overall 1-year success rate after a single CAB procedure was $70 \%$. The 1-year outcome was: $83 \%, 70 \%, 60 \%, 40 \%$ and $29 \%$ in patients with ALARMEc risk score: 0, 1, 2, 3 and 5, respectively. Total rate of complications was 11\%, including transient phrenic nerve palsy in $9.5 \%$ of cases.

Conclusions: Multi-catheter approach was needed in $21 \%$ of patients to achieve acute pulmonary vein isolation. Patients with low $(\leq 1) A L A R M E c$ risk score, preferably young individuals with sole paroxysmal $A F(A L A R M E c=0)$, are best candidates for $C B A$ procedure. Performing $C B A$ in patients with higher (>2) ALARMEc risk score should be avoided. Phrenic nerve palsy was a transient complication. (Cardiol J 2015; 22, 2: 194-200)

Key words: atrial fibrillation, cryoballoon ablation, outcome, selection of patients

\section{Introduction}

The increase in number of catheter ablation of atrial fibrillation (AF) continues around the world [1]. The time-consuming, point-by-point radiofrequency ablation technique has been challenged by using novel technologies, like cryoballoon abla- tion $(\mathrm{CAB})$, aiming for a tantalizing "single-shot" approach to facilitate and speed up the invasive procedure [2]. The cryoballoon technology is limited to pulmonary vein isolation (PVI) and previous reports showed worse outcome in patients with non-paroxysmal atrial fibrillation (NPAF) accompanied by other comorbidity. Moreover, obtaining

Address for correspondence: Prof. Thomas Neumann, Department of Cardiology, Kerckhoff Heart and Thorax Center, Benekestr. 2-8, 61231 Bad Nauheim, Germany, tel:+49 6032/996-2202, fax: + 49 6032/996-2298,

e-mail: t.neumann@kerckhoff-klinik.de

Received: 29.03.2014 Accepted: 11.11.2014 
Table 1. Baseline characteristics $(n=478)$. Continuous data were described as median, lowest (interquartile range [IQR] 25) and highest (IQR75) quartiles.

\begin{tabular}{|c|c|}
\hline Male & $244(64.55 \%)$ \\
\hline Age [years], median (IQR) & $58(49 ; 65)$ \\
\hline History of atrial fibrillation [years], median (IQR) & $5.27(2.16 ; 10.00)$ \\
\hline Paroxysmal atrial fibrillation & $320(84.65 \%)$ \\
\hline Body mass index $\left[\mathrm{kg} / \mathrm{m}^{2}\right]$, median (IQR) & $26.71(24.51 ; 29.55)$ \\
\hline \multicolumn{2}{|l|}{ Echocardiography: } \\
\hline Left atrium short diameter [mm], median (IQR) & $38(36 ; 40)$ \\
\hline Left atrium long diameter [mm], median (IQR) & $51(47 ; 55)$ \\
\hline Left atrium area $[\mathrm{mm}]$, median (IQR) & $19.24(17.28 ; 22.04)$ \\
\hline Normalized left atrium area, median (IQR) & $9.54(8.44 ; 10.82)$ \\
\hline Left ventricular ejection fraction [\%], median (IQR) & $62(57 ; 62)$ \\
\hline \multicolumn{2}{|l|}{ Medical history: } \\
\hline Glomerular filtration rate $[\mathrm{mL} / \mathrm{min}]$, median (IQR) & $92.1(80.30 ; 106.52)$ \\
\hline Hypertension & $223(58.99 \%)$ \\
\hline Coronary artery disease & $26(6.88 \%)$ \\
\hline Diabetes mellitus & $19(5.03 \%)$ \\
\hline Metabolic syndrome & $131(34.66 \%)$ \\
\hline \multicolumn{2}{|l|}{ Antiarrhythmic drugs at inclusion: } \\
\hline Class I & $180(47.62 \%)$ \\
\hline Class II & $240(63.49 \%)$ \\
\hline Class III & 95 (25.13\%) \\
\hline Amiodarone & $39(10.32 \%)$ \\
\hline Dronedaron & $30(7.94 \%)$ \\
\hline Sotalol & $26(6.88 \%)$ \\
\hline
\end{tabular}

satisfactory effects in case of variant anatomy of pulmonary veins (PVs) can be challenging with the need for additional "touch-up" with additional catheters [3].

We hypothesized that the acute and long-term success rate after a single CBA procedure is related to proper pre-procedural selection of patients.

\section{Methods}

Between 2005 and 2012 we enrolled 378 consecutive patients (Table 1 ) with electrocardiographic (ECG)-documented AF on at least 2 ECGs in last 3 months, symptomatic AF, drug-refractory $\mathrm{AF}$, age $\geq 18$ and $\leq 80$ years, signed informed consent. Exclusion criteria were as follows: reversible cause of AF (such as hyperthyroidism), moderateto-severe valvular stenosis or insufficiency, previous myocardial infarction, any congenital heart disease, left ventricular ejection fraction $<50 \%$, coronary artery bypass graft surgery within the last 3 months, chronic obstructive pulmonary disease treated with beta-sympathomimetic drugs, severe respiratory insufficiency, known bleeding diathesis, intolerance of heparin or oral anticoagulation, attempted AF ablation in the past, left atrial thrombus, pregnancy or breastfeeding, New York Heart Association class IV, severe comorbidity, abuse of drugs of alcohol, the diameter of any PV or common ostium $>27 \mathrm{~mm}$ in magnetic resonance imaging, no signed informed consent.

\section{Combined ALARMEc risk score}

The ALARMEc (Atrial fibrillation type, Left Atrium size, Renal insufficiency, Metabolic syndrome, cardiomyopathy) combined risk score was calculated using the formula (Table 2), where Bi is regression coefficient by significant predictor $\mathrm{Xi}$ (all predictors were dichotomized) in Cox regressions model. For simple usage the calculated ALARMEc risk score was rounded off to the nearest integer.

The combined ALARMEc risk score was proved to be an effective tool for discrimination of patients outcome after any ablation procedure $[4,5]$.

We calculated ALARMEc risk score for each individual patient. 
Table 2. Calculation of the ALARMEc risk score.

\begin{tabular}{llcc}
\hline \multicolumn{5}{c}{ ALARMEc $\left.=\frac{\exp \left(\sum \mathrm{BiXi}\right)}{1+\exp \left(\sum \mathrm{BiXi}\right)}-0.5\right)^{*}-10$} & $\mathbf{B i}$ \\
\hline \multicolumn{2}{l}{ ALARMEc risk score } & Non-proxymal AF & -0.611 \\
\hline A & Type of atrial fibrillation & $\mathrm{NLA} \geq 11.5$ & -0.366 \\
LA & Size of left atrium & GFR $<68 \mathrm{~mL} / \mathrm{min}$ & -0.398 \\
R & Renal function & Yes & -0.324 \\
ME & Metabolic syndrome & Yes & -0.445 \\
\hline
\end{tabular}

AF — atrial fibrillation; GFR — glomerular filtration rate; NLA — normalized left atrium area

\section{Pre-ablation}

Medical history was obtained during ambulatory visits with a thorough review of the medical records including ECGs and Holter-ECG recordings showing episodes of AF. All patients gave written informed consent. The study was approved by the local institutional Ethics Committee. Oral anticoagulation was stopped 3 days before intervention and replaced by subcutaneous low-molecular-weight heparin. Antiarrhythmic drugs were discontinued at least 3 days before ablation. Beta-blockers were allowed according to the protocol.

All patients underwent echocardiography to determine left atrium (LA) diameter and exclude LA thrombus. The LA size was assessed by measurement of short and long axis in the apical 4 -chamber view.

\section{Intervention and post-ablation management}

The procedure was previously described $[3,6]$. After the transseptal punctures were performed, heparin was introduced with the aim of keeping the activation clotting time $>300 \mathrm{~s}$ throughout the whole procedure. Briefly, we used 23- and/or 28-mm cryoballoon (Arctic Front ${ }^{\mathrm{TM}}$, MedtronicCryocath). The single application time was 240-300 s. During CBA of the right-sided PVs, unaffected phrenic movement was monitored by both continuous phrenic nerve stimulation and continuous monitoring of spontaneous breathing. An observation period after initial isolation to check for recurrence of PV conduction was 30 min. If PVI could not be achieved with a firstchoice cryoballoon size, we additionally used the different sized one. If PVI could not be confirmed after 5 consecutive applications per $\mathrm{PV}$ with any balloon, an 8-mm tip cryoablation catheter (FreezorMAX $^{\mathrm{TM}}$, MedtronicCryocath) was used for touch-up ablation to complete PVI which was verified as complete elimination of all PV signals at the antral or ostial level. Additionally, exit and entrance-block of all PVs were confirmed by pacing maneuvers, as previously described.

After the procedure, intravenous heparin was continued to achieve a partial thromboplastin time of $60-80 \mathrm{~s}$, followed by oral anticoagulation with coumadin, for at least 3 months, targeting an international normalized ratio of 2-3. Antiarrhythmic treatment was stopped. Beta-blockers were allowed during the follow-up.

\section{Follow-up}

Our strict follow-up protocol fulfills latest recommendations [7]. After discharge from the hospital, patients were scheduled for quarterly follow-up visits. Late follow-up ( $>1$ year post-intervention) was performed once a year. Seven-day Holter-ECG recordings were obtained at each follow-up visit. Each patient, in case of any palpitations, was instructed to have ECG performed for confirming or excluding atrial fibrillation, atrial flutter or atrial tachycardia (AFLAT).

\section{Statistical analysis}

The study was designed as an observational cohort study with the analyzed period of one year. The end-point of the study was the first AFLAT-recurrence after the 3-month blanking period in the 1-year follow-up since the index procedure, in the absence of antiarrhythmic drugs (class I and III) therapy. Kaplan-Meier univariate analysis was used to estimate AFLAT-free survival. Continuous data were described as median, lowest (IQR25) and highest (IQR75) quartiles. The discrete variables were given in number and percentage. The differences were considered significant be error probability $\mathrm{p}<0.05$. 
Table 3. Acute isolation of pulmonary veins (PVs) related to PVs anatomy and type and number of catheter used $(p=0.149)$. Consequently, 1,860 out of $1,871(99.4 \%)$ PVs were isolated with singleor multi-catheter approach.

\begin{tabular}{|c|c|c|c|}
\hline & $\begin{array}{c}\text { All patients } \\
N=378(100 \%)\end{array}$ & $\begin{array}{c}\text { Typical PVs anatomy } \\
\text { N }=341(90.21 \%)\end{array}$ & $\begin{array}{c}\text { Atypical PVs anatomy } \\
\qquad N=37(9.79 \%)\end{array}$ \\
\hline Single balloon approach: & $300(79.36 \%)$ & $270(74.07 \%)$ & $30(7.93 \%)$ \\
\hline 23-mm CBA & $52(13.76 \%)$ & $51(13.49 \%)$ & $1(0.26 \%)$ \\
\hline 28-mm CBA & $248(65.61 \%)$ & $219(57.93 \%)$ & $29(7.67 \%)$ \\
\hline Multi-catheter approach* & $78(20.63 \%)$ & $71 * *(18.78 \%)$ & $7(1.85 \%)$ \\
\hline
\end{tabular}

*Additional catheter (other sized cryoballoon and/or FreezorMax ${ }^{\mathrm{TM}}$ ) was needed, including $29(7.67 \%)$ in whom both two sized balloons and FreezorMax ${ }^{\mathrm{TM}}$ was used; ${ }^{* *} 45$ patients with typical anatomy but critical pulmonary veins angulation; CAB - cryoballoon ablation

Table 4. The incidence of complications.

\begin{tabular}{lccccccc}
\hline $\mathbf{N}=378$ & Tamponade & Pericardial effusion & TIA & Stroke & PNP & All complications & Total \\
\hline $\mathrm{N}(\%)$ & $2(0.6 \%)$ & $2(0.6 \%)$ & $1(0.3 \%)$ & $1(0.3 \%)$ & $36(9.5 \%)$ & $42(11.1 \%)$ & $\mathbf{3 7 8}(\mathbf{1 1 . 1} \%)$ \\
\hline
\end{tabular}

TIA — transient ischemic attack; PNP — phrenic nerve palsy

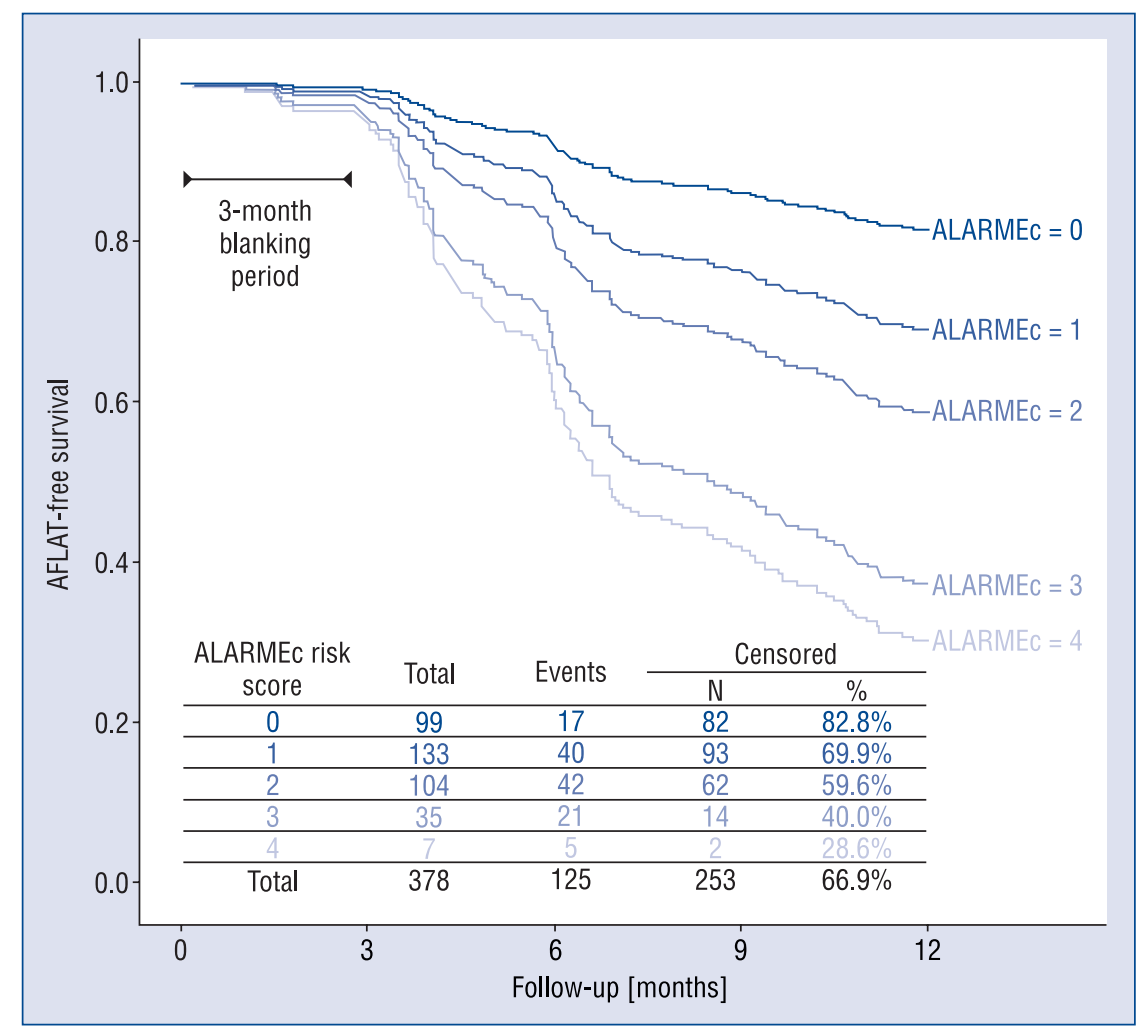

Figure 1. The 1-year outcome after a single cryoballoon ablation procedure related to patients' ALARMEc (Atrial fibrillation type, Left Atrium size, Renal insufficiency, Metabolic syndrome, cardiomyopathy) risk score; AFLAT — atrial fibrillation, atrial flutter or atrial tachycardia.

\section{Results}

In total, 378 consecutive patients were treated (Table 3$)$. Consequently, 1,502 out of 1,512 (99.3\%) $\mathrm{PVs}$ were isolated. The median procedure and fluoroscopy time was $3.8 \mathrm{~h}(3.0 ; 4.5)$ and $37.8 \mathrm{~min}$ (27.0;55.2), respectively.

Peri-procedural complications are presented in Table 4. Right phrenic nerve palsy (PNP) was observed in $36(9.5 \%)$ patients during cryoablation 
of the right superior PV. PNP resolved in 14 cases during the procedure. An early termination of cryoenergy application during right phrenic pacing did not prevent the subsequent occurrence of PNP. In all other patients, full re-covery of right phrenic function was observed during 1-year follow-up. We observed higher occurrence of PNP in patients treated with the $23 \mathrm{~mm}$ balloon as compared with $28 \mathrm{~mm}: 24$ vs. 12 patients, respectively.

The overall 1-year success rate after a single $\mathrm{CAB}$ procedure was $66.9 \%$. The 1 -year outcome related to calculated ALARMEc risk score is presented in Figure 1. The most favorable 1-year outcome was observed in patients with ALARMEc scores 1 and $2(83 \%$ and $70 \%$ AFLAT-free, respectively). On the contrary, individuals with lowest ALARMEc scores (3 and 4; 40\% and 29\% AFLAT-free, respectively) should not be rather the candidates for $\mathrm{CAB}$.

\section{Discussion}

The cryoballoon technology is limited to PVI. Our results show that adequate pre-procedural selection of patients and focusing on individuals with low ALARMEc risk score, is crucial both for acute PVI and long-term outcome after CBA procedure.

\section{Acute success}

The isolation of PVI is a cornerstone of CBA. Previous analysis showed that acute PVI, with a single cryoballoon catheter only, could be completed in $78 \%$ of patient [8]. Better acute results were reported in studies in which additional cryoballoon catheter $(98.7 \%)$ or concomitant focal ablation (98.9\%) was used [8]. Our results are comparable. We were able to isolate all PVs in $79 \%$ of patients with a single balloon only. Additional catheter allowed for PVI in 99.3\%.

Pre-procedural imaging of PVs anatomy seems to be crucial, especially for centers restricted to 1 cryoballoon catheter only. Failure in acute PVI could result not only in AF-recurrence but also in LA tachycardia related to CBA [9, 10]. Mikhaylov et al. [9] showed that atrial tachycardia after CBA was more frequently observed in patients in whom procedure was more laborious (atypical anatomy and need for additional "touch-up" lesions) [10].

Recently Goginn et al. [11] has showed that the mechanisms leading to PVs conduction recovery were difficulty in correct positioning of the cryoballoon and geometrical mismatch between the cryoballoon and the PV ostium. The good cryoballoon-tissue contact is an important parameter influencing the cryoenergy-related lesion. This parameter is likely involved in the PV reconduction process, especially at PV with difficult geometries [11]. Optimization of tissue contact may require utilization of technical maneuvers [11, 12], different sized balloons $[3,6,8,13]$, and "touch-up" focal lesions [3, 6, 8, 13-15]. Moreover, peri-procedural PNP results in abrupt interruption of cryoenergy application by an operator which can be the next cause of not-complete acute PVI success. Still, in our series, we have not found any differences in outcome comparing patients with and without transient PNP during CBA procedure.

\section{ALARMEc risk score and long-term success}

In 1998, Haissaguerre et al. [16] showed that PVs have been the crucial source of the triggers that initiate AF and electrical PVI could be an effective therapeutic option for AF. Currently we know that AF has a tendency to becoming more persistent over time [17]. The progression of electrical and further structural remodeling of atria promotes both reentry and ectopic activity which can serve not only as substrate but also as triggers for AF [17]. Sole PVI has low effectiveness in such electrically and structurally changed environment.

Many factors (type of AF, LA size, age, sleep apnea and obesity, hypertension, LA fibrosis detected by magnetic resonance imaging, diabetes, a rise in right atrium magnetic strength) have been proposed as predictors of a poorer outcome after PVI $[18,19]$ but the discrepancies among authors [20-25] showed the complicated nature of already ablated atrial tissue. We applied a combined ALARMEc risk score which effectively discriminates patient outcome after any ablation procedure [4, 5]. Our data show that patients with low $(\leq 1)$ ALARMEc risk score have most favorable outcome. In individuals with high $(\geq 3)$ ALARMEc risk score the outcome is poor.

There are many possible reasons that patients with low $(\leq 1)$ ALARMEc risk score may have such a good outcome. This group has not-enlarged atria and paroxysmal form of AF. The probability of deep remodeling process, in such settings, is low and the development of AF is rather trigger- and not substrate-dependent. Most triggers originate in LA-PVs junction [26]. PVI-focused CBA, which eliminates triggers, and partially substrate located near LA-PVs junction, results in high success rate in such patients. Failure of PVI in this group is rather a result of incomplete isolation of LA-PVs triggers or existing non-LA-PVs triggers. Moreover, the combination of normal-sized LA and relatively big 
balloon may result not only in ostial circumferential but also in PVI by ipsilateral antral block ablation, which might further improve the clinical outcome [27]. Comparable good results in similar low risk patients, were recently showed by others [15, 28]. Moreover, CBA procedure was proposed as first-line therapy in patients with lone paroxysmal AF [15]. Indeed, our results show that it can be a case for patients with lowest $(=0)$ ALARMEc risk score.

Patients with moderate $(=2)$ ALARMEc risk score are at the borderline of persistency of AF. The ablation procedure should be advocated in this group to interrupt a process of continuous remodeling. We show that PVI with CBA will have moderate 1-year success rate. More invasive approach, with additional lesions within atria, should be rather considered to further improve the outcome in this cohort.

Patients with high $(=3)$ and very high $(=4)$ ALARMEc risk score, i.e. patients with non-paroxysmal AF with concomitant comorbidity which further promotes atrial remodeling, had poor 1-year outcome. Steep drop in AFLAT-free survival was noted since the first months after the blanking period (Fig. 1). Other authors also showed very poor outcome in similar cases [29]. These patients are rather candidates for radiofrequency ablation with additional substrate modifications or in severe cases for rate control only.

\section{Complications}

The incidence of major complications was comparable to previously published data [8]. We noted peri-procedural PNP in $9.52 \%$ of cases. Still, in all our patients, the full recovery from PNP, between some minutes after cessation of cryoenergy application up to 1 year after the ablation procedure, was observed. Nevertheless, despite different safety approaches proposed recently, the problem of PNP has not been eliminated [30].

\section{Clinical implications}

Firstly, selection of patients is crucial. Patients with typical anatomy and lower ALARMEc risk score appear to be the first line candidates for CBA procedure.

Secondly, more than one single cryoballoon can be needed to complete PVI, especially in patients with atypical anatomy of PVs.

Thirdly, patients should be aware of possible procedure-related complications, including PNP.

Finally, ongoing surveillance is warranted, even if CBA was deemed initially successful. In- cidence of late recurrence may be related to the extent of ECG monitoring and earlier recurrence may be missed in selected patients with no or minimal symptoms [7]. Additionally, procedure of ablation is one of many steps in the process of treating a patient with AF. Focusing on controllable risk factors, like renal insufficiency and components of metabolic syndrome, could shift a patient to a lower ALARMEc risk group and further improve the outcome.

\section{Limitations of the study}

This is a single-center, observational cohort report with the inherent limitations of this study design. However, there was no selection bias for study inclusion since all consecutive patients undergoing cryoballoon ablation procedure were included for analysis.

The presented data reflect our experience with cryoballoon technique gained in a high volume center by operators already experienced in standard and advanced ablation procedures. The results should not be extrapolated to low-volume centers and unexperienced operators.

\section{Conclusions}

Cryoballoon ablation, aimed at PVI, allowed to achieve satisfactory outcome in the selected population of patients.

Acute single-cryoballoon PVI success was high $(79 \%)$ but not complete. Multi-catheter approach was needed in $78(20.6 \%)$ patients.

Patients with low $(\leq 1)$ ALARMEc risk score, preferably young individuals with sole paroxysmal AF (ALARMEc $=0$ ), are best candidates for CBA procedure. Contrary, performing CBA in patients with high ALARMEc risk score should be avoided.

PNP was a transient complication. Other serious complication with the need for intervention were relatively rare.

Conflict of interest: Doctor Maciej Wójcik was supported by European Heart Rhythm Association (2-years Clinical Electrophysiology Fellowship, 2007-2009); Professor Thomas Neumann has received speakers' honoraria from MedtronicCryocath; Doctor Malte Kuniss has received speakers' honoraria and for advisory board meetings from MedtronicCryocath; Doctor Heinz-Friedrich Pitschner has received honoraria for advisory board meetings from MedtronicCryocath. 


\section{References}

1. Cappato R, Calkins H, Chen SA et al. Updated worldwide survey on the methods, efficacy, and safety of catheter ablation for human atrial fibrillation. Circulation Arrhythmia Electrophysiology, 2010; 3: 32-38.

2. Ernst S. Cryo balloon pulmonary vein isolation: Is it really all so "simple"? J Am Coll Cardiol, 2013; 61: 1724-1725.

3. Neumann T, Vogt J, Schumacher B et al. Circumferential pulmonary vein isolation with the cryoballoon technique results from a prospective 3-center study. J Am Coll Cardiol, 2008; 52: 273-278.

4. Berkowitsch A, Kuniss M, Greiss H et al. Impact of impaired renal function and metabolic syndrome on the recurrence of atrial fibrillation after catheter ablation: A long term follow-up. Pacing Clin Electrophysiol, 2012; 35: 532-543.

5. Wojcik M, Berkowitsch A, Greiss H et al. Repeated catheter ablation of atrial fibrillation. Circ J 2013; 77: 2271-2279.

6. Neumann T, Wojcik M, Berkowitsch A et al. Cryoballoon ablation of paroxysmal atrial fibrillation: 5-year outcome after single procedure and predictors of success. Europace, 2013; 15: 1143-1149.

7. Calkins H, Kuck KH, Cappato R et al. 2012 HRS/EHRA/ECAS Expert Consensus Statement on Catheter and Surgical Ablation of Atrial Fibrillation: Recommendations for patient selection, procedural techniques, patient management and follow-up, definitions, endpoints, and research trial design. Europace, 2012; 14: 528-606.

8. Andrade JG, Khairy P, Guerra PG et al. Efficacy and safety of cryoballoon ablation for atrial fibrillation: A systematic review of published studies. Heart Rhythm, 2011; 8: 1444-1451.

9. Mikhaylov EN, Bhagwandien R, Janse PA, Theuns DA, Szili-Torok T. Regular atrial tachycardias developing after cryoballoon pulmonary vein isolation: Incidence, characteristics, and predictors. Europace, 2013; 15: 1710-1717.

10. Wojcik M, Berkowitsch A, Pitschner HF, Kuniss M, Neumann T. Atrial tachycardia after cryoballoon ablation of paroxysmal atrial fibrillation. Europace, 2014; 16: 306.

11. Godin B, Savoure A, Gardey K, Anselme F. Lessons from radiofrequency redo-procedure after cryoballoon pulmonary vein isolation for paroxysmal atrial fibrillation. Circ J, 2013; 77: 2009-2013.

12. Chun KR, Schmidt B, Metzner A et al. The 'single big cryoballoon' technique for acute pulmonary vein isolation in patients with paroxysmal atrial fibrillation: A prospective observational single centre study. Eur Heart J, 2009; 30: 699-709.

13. Vogt J, Heintze J, Gutleben KJ, Muntean B, Horstkotte D, Nolker G. Long-term outcomes after cryoballoon pulmonary vein isolation: Results from a prospective study in 605 patients. J Am Coll Cardiol, 2012; 61: 1707-1712.

14. Packer DL, Kowal RC, Wheelan KR et al. Cryoballoon Ablation of Pulmonary Veins for Paroxysmal Atrial Fibrillation: First Results of the North American Arctic Front (STOP AF) Pivotal Trial. J Am Coll Cardiol 2013; 61: 1713-1723.

15. Namdar M, Chierchia GB, Westra S et al. Isolating the pulmonary veins as first-line therapy in patients with lone paroxysmal atrial fibrillation using the cryoballoon. Europace, 2012; 14: 197-203.

16. Haissaguerre M, Jais P, Shah DC et al. Spontaneous initiation of atrial fibrillation by ectopic beats originating in the pulmonary veins. N Engl J Med, 1998; 339: 659-666.
17. Xu Y, Sharma D, Li G, Liu Y. Atrial remodeling: New pathophysiological mechanism of atrial fibrillation. Med Hypotheses, 2013; 80: 53-56.

18. Calkins H, Kuck KH, Cappato R et al. 2012 HRS/EHRA/ECAS expert consensus statement on catheter and surgical ablation of atrial fibrillation: Recommendations for patient selection, procedural techniques, patient management and follow-up, definitions, endpoints, and research trial design. J Interv Card Electrophysiol, 2012; 33: 171-257.

19. Sato $\mathrm{Y}$, Yoshida K, Ogata $\mathrm{K}$ et al. An increase in right atrial magnetic strength is a novel predictor of recurrence of atrial fibrillation after radiofrequency catheter ablation. Circ J, 2012; 76: $1601-1608$.

20. Shah AN, Mittal S, Sichrovsky TC et al. Long-term outcome following successful pulmonary vein isolation: Pattern and prediction of very late recurrence. J Cardiovasc Electrophysiol, 2008; 19: 661-667.

21. Chao TF, Tsao HM, Lin YJ et al. Clinical outcome of catheter ablation in patients with nonparoxysmal atrial fibrillation: Results of 3-year follow-up. Circulation Arrhythmia Electrophysiology, 2012; 5: 514-520.

22. McCready JW, Smedley T, Lambiase PD et al. Predictors of recurrence following radiofrequency ablation for persistent atrial fibrillation. Europace, 2011; 13: 355-361.

23. Rostock T, Salukhe TV, Steven D et al. Long-term single- and multiple-procedure outcome and predictors of success after catheter ablation for persistent atrial fibrillation. Heart Rhythm, 2011; 8: 1391-1397.

24. Hunter RJ, Ginks M, Ang R et al. Impact of variant pulmonary vein anatomy and image integration on long-term outcome after catheter ablation for atrial fibrillation. Europace, 2010; 12: 1691-1697.

25. D'Ascenzo F, Corleto A, Biondi-Zoccai G et al. Which are the most reliable predictors of recurrence of atrial fibrillation after transcatheter ablation? A meta-analysis. Int J Cardiol, 2012; 167: 1984-1989.

26. Weerasooriya R, Khairy P, Litalien J et al. Catheter ablation for atrial fibrillation: Are results maintained at 5 years of follow-up? J Am Coll Cardiol, 2011; 57: 160-166.

27. Arentz T, Weber R, Burkle G et al. Small or large isolation areas around the pulmonary veins for the treatment of atrial fibrillation? Results from a prospective randomized study. Circulation, 2007; 115: 3057-3063.

28. Van Belle Y, Janse P, Theuns D, Szili-Torok T, Jordaens L. One year follow-up after cryoballoon isolation of the pulmonary veins in patients with paroxysmal atrial fibrillation. Europace, 2008; 10: 1271-1276.

29. Kojodjojo P, O'Neill MD, Lim PB et al. Pulmonary venous isolation by antral ablation with a large cryoballoon for treatment of paroxysmal and persistent atrial fibrillation: Medium-term outcomes and non-randomised comparison with pulmonary venous isolation by radiofrequency ablation. Heart, 2010; 96: 1379-1384.

30. Kuhne M, Knecht S, Altmann D et al. Phrenic nerve palsy during ablation of atrial fibrillation using a $28-\mathrm{mm}$ cryoballoon catheter: Predictors and prevention. J Interv Card Electrophysiol, 2013; 36: 47-54. 